\title{
What can the National Cancer Database tell us about disparities in advanced bladder cancer outcomes?
}

\author{
Shreyas S. Joshi ${ }^{1}$, Elizabeth R. Handorf ${ }^{2}$, Marc C. Smaldone ${ }^{1}$, Daniel M. Geynisman ${ }^{3}$ \\ ${ }^{1}$ Division of Urologic Oncology, Department of Surgical Oncology, ${ }^{2}$ Department of Bioinformatics and Biostatistics, ${ }^{3}$ Department of Hematology/ \\ Oncology, Fox Chase Cancer Center, Philadelphia, PA, USA \\ Correspondence to: Daniel M. Geynisman, MD. Department of Hematology/Oncology, Fox Chase Cancer Center, Temple University Health System, \\ 333 Cottman Avenue, Philadelphia, PA 19111, USA. Email: daniel.geynisman@fccc.edu. \\ Provenance: This is a Guest Editorial commissioned by Section Editor Xiao Li (Department of Urology, Jiangsu Cancer Hospital, Jiangsu Institute of \\ Cancer Research, Nanjing Medical University Affiliated Cancer Hospital, Nanjing, China). \\ Comment on: Weiner AB, Keeter MK, Manjunath A, et al. Discrepancies in staging, treatment, and delays to treatment may explain disparities in \\ bladder cancer outcomes: An update from the National Cancer Data Base (2004-2013). Urol Oncol 2018;36:237.e9-237.e17.
}

Submitted Jun 11, 2018. Accepted for publication Jun 20, 2018.

doi: $10.21037 /$ tau.2018.06.16

View this article at: http://dx.doi.org/10.21037/tau.2018.06.16

\section{Introduction}

Advanced bladder cancer is largely a lethal disease that has demonstrated little improvement in survival trends over the past 50 years. Five-year survival for advanced bladder cancer has remained at about $8 \%$ since 1973 ; though, as in most diseases, survival outcomes are not evenly distributed among different sociodemographic groups (1).

Disparities in bladder cancer outcomes by race, ethnicity, gender, and socioeconomic status (SES) have repeatedly been demonstrated using large retrospective databases (2-4). In a recent issue of Urologic Oncology: Seminars and Original Investigations, Weiner et al. added to this literature by executing a well-designed retrospective analysis on advanced bladder cancer patients between 2004-2013 using the National Cancer Database (NCDB) (5). They analyzed over 320,000 bladder cancer patients in the United States, specifically focusing on the $7.6 \%(n=25,000)$ that presented with late stage disease, defined as AJCC stage III or stage IV. Using logistic regression models, treatment and sociodemographic variables were queried with respect to survival outcomes and delays in treatment.

The study found that female sex $(\mathrm{HR}=1.23)$, black race $(H R=1.51)$, Hispanic ethnicity $(H R=1.12)$, lower income ( $\mathrm{HR}=1.23$ for the lowest 4th quartile income), and educational regions $(\mathrm{HR}=1.23$ for the lowest 4 th quartile education) were associated with increased odds of presenting with late stage disease. For overall survival, female sex $(H R=1.16)$, black race $(H R=1.10)$, and lower regional income levels $(\mathrm{HR}=1.08$ for the lowest 4 th quartile) were associated with poorer survival. Total of 13,018 patients had information related to time between diagnosis and first treatment. Men were more likely than women to have a treatment delay $>12$ weeks $(13.5 \%$ vs. $12 \%)$. Black (18.4\%) and Hispanic (19.2\%) patients were more likely to have treatment delays compared to white patients (12.7\%). Patients living in regions with the lowest income and education quartiles, and patients with Medicaid/ No insurance were all marginally more likely to experience treatment delays.

Unfortunately, it should come as no surprise at this point that minorities and women with advanced bladder cancer have worse outcomes (note that although the NCDB only provides overall survival, given the advanced stage of this cohort, it is assumed to be a good surrogate for cancerspecific survival). Multiple retrospective studies on gender differences in bladder cancer, for example, have persistently shown outcome differences for all stages (6). For patients presenting with pT4a disease, May et al. demonstrated a dramatically lower 5 -year cancer-specific survival for women $v$ s. men $(15 \%$ vs. $35 \%, \mathrm{P}=0.003)$ (7). With the consistency of these findings, we must aim to delve further into the mechanisms that fuel such outcome differences.

Although the NCDB has a number of well described limitations (8) and the authors analyzed both stage III and 
IV patients together (groups that can exhibit vastly different outcomes), Weiner et al. should be commended for helping shed light on the magnitude of sociodemographic disparities in bladder cancer outcomes. The study is unique in evaluating variables associated with stage presentation and survival outcomes for only late stage patients. The current study is also notable within historical context: SEER data on bladder cancer patients between 1990-2005 demonstrated worse survival for women and African Americans (9). Sadly, these discrepancies have yet to be adequately addressed.

As clinicians and researchers, we should, of course, aim to eliminate disparities in cancer outcomes; but before getting to that step, we must trust that we have an accurate representation of the true variation in the population. The NCDB is a powerful tool, but its data has well-documented limitations; health services researchers have recently addressed "best practices" while using the database (10). Below, we discuss some of the potential advantages, as well as the pitfalls and difficulties in using the NCDB for bladder cancer disparities research.

\section{Using the NCDB for disparities research}

Understanding bladder cancer disparities is fundamentally a population-based query, which relies on population-based data. The NCDB is a hospital-based registry that selects cases based on hospital participation [Committee on Cancer $(\mathrm{CoC})$ accreditation]. This accounts for approximately $30 \%$ of hospitals in the United States. There are differences in the number and density of $\mathrm{CoC}$-accredited hospitals throughout the United States, leading to differences by region of who gets treated at an NCDB-reporting hospital. Some states are over-represented and some are underrepresented (89\% of patients are captured in Delaware vs. $27 \%$ in Arizona, for example); and these variations inherently carry the risk of selecting an unrepresentative sample of the population (11). Further, sociodemographic factors themselves may influence whether a patient seeks care at a CoC-accredited institution. SEER, in contrast, is a population-based registry that includes cases based on geographic location rather than hospital accreditation. These locations were carefully selected to be representative of the U.S. population; and even though SEER only captures $30 \%$ of cancer diagnoses ( $v s .70 \%$ for the NCDB), it may be more appropriate to prefer its use over the NCDB for detailing sociodemographic disparities (8). As a striking example of how this impacts disparities research, Bleicher et al. evaluated disparities in breast cancer treatment and survival outcomes between SEER and NCDB registries. They showed that although both sets of data produced similar trends, there were differences in which specific variables were deemed significant (12). The current study should therefore be evaluated in the context of the existing literature on bladder cancer disparities.

A database's ability to accurately describe outcomes disparities is predicated on the assumption that sociodemographic variables collected in the database actually reflect patient characteristics. A weakness of the NCDB is that income and education, which are two of the most powerful variables that determine SES, are abstracted from zip-code level census data, and then collapsed into quartiles. The data therefore lacks sufficient granularity to precisely analyze how individual-level differences in income or education level impact treatment and survival outcomes.

The number of cases available in the NCDB is a major benefit when studying disparities, but it also presents challenges that are often overlooked. With the large number of patients available, one can easily fit multiple regression models to identify the independent effects of the various sociodemographic variables, which may be highly correlated. One can also successfully study relatively rare outcomes (such as diagnosis of advanced bladder cancer), and investigate interaction effects of interest (as Weiner et al. examined whether treatment effects differed by sociodemographic). However, the large number of cases also can lead to highly statistically significant results for small differences, which may not be clinically meaningful. Researchers should therefore be careful to pay attention to the magnitude of the effects, not just the significance. Furthermore, increasing the sample size decreases the variability of the estimates, but it does not decrease potential biases. Retrospective, non-randomized studies are always subject to biases from unmeasured confounders. For example, in the case of bladder cancer, smoking behavior may be an important factor associated with both sociodemographic variables and cancer outcomes.

Perhaps most importantly, the mechanisms through which disparities lead to worse outcomes is virtually impossible to understand in large registries. Why, in the analysis by Weiner et al., the fact that SES remains a significant factor in predicting staging and late-stage survival even when controlling for related factors is hard to explain. Can merely being poor and underprivileged be the mechanism driving discrepant outcomes? Is the biology of bladder cancer different based on SES though a lifetime of different perturbations and epigenetics? Perhaps—but 
there is probably an unmeasured mix of important factors that are the bulk of the true mechanism driving these disparities. Explaining such disparities is an entire field unto itself, though integrating data acquired from registries with existing conceptual models of health disparities may eventually help strengthen our understanding of the mechanisms and causes fueling the disparities (13).

The types of treatment each patient receives is clearly an important factor in determining outcomes, but it is often difficult to know how much each treatment is affecting outcomes. The NCDB captures the first treatment after diagnosis, but only if the first treatment occurred at a reporting institution. Thus, if a patient is diagnosed at a local non-CoC hospital and begins treatment there for localized bladder cancer, but then moves to a $\mathrm{CoC}$ hospital after their bladder cancer progresses to advanced disease, that patient will not be captured by the NCDB. Similarly, patients traveling between facilities may not have neoadjuvant or adjuvant therapies recorded. Importantly, the NCDB is unable to report on subsequent or follow-up treatments. It was therefore appropriate for Weiner $e t a l$. to study time to first treatment as a quality metric that differed between populations, as this metric may be a more powerful sign of which patients are actually receiving appropriate treatment. It is conceivable that inclusion in clinical trials or management at certain centers of excellence are associated with better outcomes, regardless of sociodemographic background; but such variables are either absent or insufficiently robust for appropriate analysis.

Despite its shortcomings, the NCDB has multiple advantages that have made it a boon to large-scale cancer research. The NCDB contains over 80 de-identified data variables describing sociodemographic, disease, treatment, and follow-up characteristic. A specific strength of the NCDB is the availability of treatment data; more data on types and timing of treatment is available through the NCDB than is present in the SEER database. Although the NCDB only acquires information from $30 \%$ of U.S. hospitals, it captures $70 \%$ of all newly-diagnosed cancer cases, far in excess of both SEER (30\%) and SEERMedicare (12\%) registries. Although SEER-Medicare can classify comorbidities through the use of ICD-9 diagnosis codes, the NCDB has comorbidity data on a far larger number of patients, albeit using the modified CharlsonDeyo comorbidity score. It is also important to note that the NCDB has a shorter lag-time between diagnosis and data availability: 2 years for treatment and 3 years for vital statistics, vs. 3 years for all data in SEER and every other year from SEER-Medicare during linkage years (8). The NCDB is therefore able to provide a more contemporary view of disease trends. For bladder cancer, a disease that has seen a huge increase in treatment options over the past few years, it is implicitly advantageous to have more contemporary (and more rapidly updated) data.

\section{Conclusions}

Weiner et al., using the NCDB, showed that disparities in late-stage presentation and survival in bladder cancer continue to manifest along sociodemographic lines. The research is important to help bring attention to these likely avoidable disparities in bladder cancer outcomes. We should, nonetheless, be cautious in over-interpreting such trends based on retrospective data. Prospective data often undermines our initial assumptions regarding drivers of improved survival. Prostate cancer is a prime example; Halabi et al. recently presented pooled data from nine prospective trials of docetaxel in advanced prostate cancer, and showed that African American men actually demonstrated better overall survival than Caucasian men (14).

Although the NCDB, like all large registries, has specific limitations, it is also a powerful tool to help define challenges related to inequitable disease prevalence, treatment trends, and cancer outcomes. Registry-based retrospective research is exploratory and hypothesisgenerating, and thus has a unique role to play within the research ecosystem. Resulting insights should ideally be used to develop prospective datasets and interventions with the aim of improving care for all patients. Finding outcome disparities is far easier than finding viable solutions to address those disparities. Keeping this in mind, it should be the goal of all disparities researchers to identify interventions that start to narrow the gap.

\section{Acknowledgements}

None.

\section{Footnote}

Conflicts of Interest: The authors have no conflicts of interest to declare.

\section{References}

1. Abdollah F, Gandaglia G, Thuret R, et al. Incidence, 
survival and mortality rates of stage-specific bladder cancer in United States: a trend analysis. Cancer Epidemiol 2013;37:219-25.

2. Jacobs BL, Montgomery JS, Zhang Y, et al. Disparities in bladder cancer. Urol Oncol 2012;30:81-8.

3. Pan K, Klapheke A, Cress R, et al. No improvement in metastatic bladder cancer health disparity over the last two decades. J Clin Oncol 2017;35:e18076.

4. Lee CT, Dunn RL, Williams C, et al. Racial disparity in bladder cancer: trends in tumor presentation at diagnosis. J Urol 2006;176:927-33; discussion 933-4.

5. Weiner AB, Keeter MK, Manjunath A, et al. Discrepancies in staging, treatment, and delays to treatment may explain disparities in bladder cancer outcomes: An update from the National Cancer Data Base (2004-2013). Urol Oncol 2018;36:237.e9-237.e17.

6. Marks P, Soave A, Shariat SF, et al. Female with bladder cancer: what and why is there a difference? Transl Androl Urol 2016;5:668-82.

7. May M, Bastian PJ, Brookman-May S, et al. Genderspecific differences in cancer-specific survival after radical cystectomy for patients with urothelial carcinoma of the urinary bladder in pathologic tumor stage T4a. Urol

Cite this article as: Joshi SS, Handorf ER, Smaldone MC, Geynisman DM. What can the National Cancer Database tell us about disparities in advanced bladder cancer outcomes? Transl Androl Urol 2018;7(4):732-735. doi: 10.21037/ tau.2018.06.16
Oncol 2013;31:1141-7.

8. Boffa DJ, Rosen JE, Mallin K, et al. Using the National Cancer Database for Outcomes Research: A Review. JAMA Oncol 2017;3:1722-8.

9. Scosyrev E, Noyes K, Feng C, et al. Sex and racial differences in bladder cancer presentation and mortality in the US. Cancer 2009;115:68-74.

10. Haider AH, Bilimoria KY, Kibbe MR. A Checklist to Elevate the Science of Surgical Database Research. JAMA Surg 2018;153:505-7.

11. Lerro CC, Robbins AS, Phillips JL, et al. Comparison of cases captured in the national cancer data base with those in population-based central cancer registries. Ann Surg Oncol 2013;20:1759-65.

12. Bleicher RJ, Ruth K, Sigurdson ER, et al. Time to Surgery and Breast Cancer Survival in the United States. JAMA Oncol 2016;2:330-9.

13. Diez Roux AV. Conceptual approaches to the study of health disparities. Annu Rev Public Health 2012;33:41-58.

14. Halabi S, Dutta S, Tangen CM, et al. Overall survival between African-American (AA) and Caucasian (C) men with metastatic castration-resistant prostate cancer (mCRPC). J Clin Oncol 2018;36:LBA5005. 